Contraband Detection with Nuclear Resonance Fluorescence: Feasibility and Impact

J. Pruet, D. Lange

January 4, 2007 
This document was prepared as an account of work sponsored by an agency of the United States Government. Neither the United States Government nor the University of California nor any of their employees, makes any warranty, express or implied, or assumes any legal liability or responsibility for the accuracy, completeness, or usefulness of any information, apparatus, product, or process disclosed, or represents that its use would not infringe privately owned rights. Reference herein to any specific commercial product, process, or service by trade name, trademark, manufacturer, or otherwise, does not necessarily constitute or imply its endorsement, recommendation, or favoring by the United States Government or the University of California. The views and opinions of authors expressed herein do not necessarily state or reflect those of the United States Government or the University of California, and shall not be used for advertising or product endorsement purposes.

This work was performed under the auspices of the U.S. Department of Energy by University of California, Lawrence Livermore National Laboratory under Contract W-7405-Eng-48. 


\section{Contraband Detection with Nuclear Resonance Fluorescence: Feasibility and Impact}

\section{J. Pruet and D. Lange}

\section{Executive Summary}

In this report we show that cargo interrogation systems developed to thwart trafficking of illicit nuclear materials could also be powerful tools in the larger fight against contraband smuggling. In particular, in addition to detecting special nuclear materials, cargo scanning systems that exploit nuclear resonance fluorescence to detect specific isotopes can be used to help find

- chemical weapons

- some drugs as well as some chemicals regulated under the controlled substances act

- precious metals

- materials regulated under export control laws

- commonly trafficked fluorocarbons

To give a sense of the range and amounts of different materials that can be quickly detected with NRF systems we show in figures 1 and 2 the detectability of a few potentially important contraband items. A more thorough discussion of detectability and a more comprehensive list of detectable items is given in the text and in tables 2 -6 .

Because of the staggering scale of contraband trafficking - the value of drugs annually smuggled into the U.S. is measured in the hundreds of billions of dollars (FIA 2001), while some two million pounds of illicit chlorofluorocarbons worth some $\$ 100 \mathrm{M}$ (EIA 2003) are annually imported - installation of broadly capable cargo scanning systems could have important economic and security implications. For this reason efforts funded by the Department of Homeland Security to improve nuclear safety could also be a great boon to customs, drug interdiction, and prevention of conventional attacks.

The cargo scanning system we examine relies on nearly monochromatic light sources that are

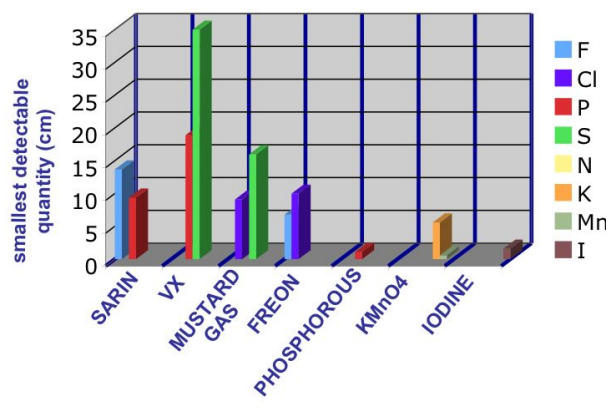

Fig. 1.- Detectability with an NRF system of constituents of some chemical weapons, Freon and chemicals regulated under the controlled substances act. The $\mathrm{y}$ axis shows the minimum linear dimension of each material in which the given constituent element of the material can be quickly detected. For example, fluorine in Sarin can be readily detected in Sarin samples larger than about $14 \mathrm{~cm}$, while the phosphorous can be detected in Sarin samples larger than about 10 $\mathrm{cm}$. These numbers correspond to a detection time of 1 second and assume the contraband is hidden in a well shielded container. The text contains a quantitative discussion of how detection times are estimated and of the detectability of many more materials.

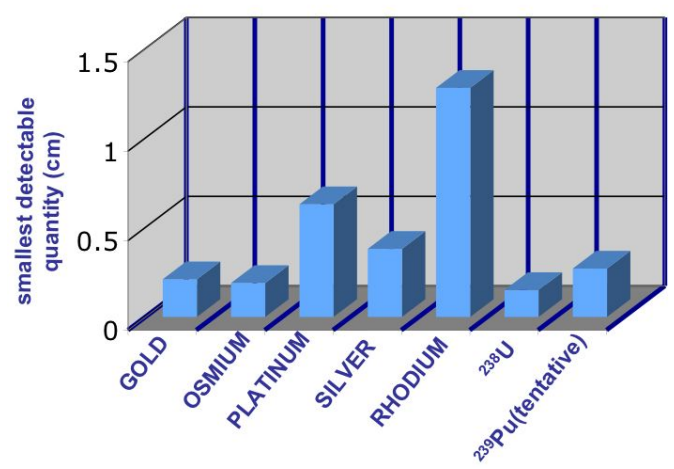

Fig. 2.- Smallest samples of different precious and fissile metals that can be quickly detected with an NRF system. Note that the result for ${ }^{239} \mathrm{Pu}$ is from a preliminary analysis of very recent experiments (Johnson 2006) and should be regarded as tentative. 
currently being developed at LLNL. These light sources are used to induce and observe nuclear resonance fluorescence (NRF), which refers to the absorption and re-emission of photons by a nucleus. Performance of these systems for the more traditional application of finding hidden uranium or plutonium has been well studied (Pruet et al. 2006; McNabb \& Pruet 2005). Relative to other methods such as those based on inducing fission with neutrons or photons, NRF systems are characterized by quick scan times and comparable or low radiological dose. However, the real strength of NRF interrogation lies in two qualitatively unique capabilities:

1. The rate at which these systems make mistakes is rigorously quantifiable and these systems cannot get tricked by clever attempts at shielding or hiding cargo.

2. NRF systems are capable of finding elements across the periodic table. From the perspective of NRF there is nothing at all special about weaponizable or radioactive materials. Uranium can be found as well as platinum, fluorine, iodine, or phosphorous or many other elements. This is not to say that every element can be easily identified through NRF. Some isotopes do not have strong low lying resonances in the $\mathrm{MeV}$ range. Nitrogen-14 is one example. It is difficult to detect such isotopes in well-shielded cargos.

Potential implications of this this second capability are the focus of this paper.

Scanning systems capable of determining the elemental content of large cargos in just a few seconds could revolutionize contraband interdiction. Observation of a substantial quantity of nitrogen in a container supposed to contain sugar would raise suspicion of drugs. Unexpected detection of fluorine and chlorine could indicate the presence of CFC's or chemical weapons. Appreciable quantities of gold, silver or platinum should never be shipped undeclared.

Later sections of this paper present a detailed account of different materials that can be quickly detected through NRF scanning. We also comment on the possible impact of being able to detect specific materials. A full study of the operational and economic impact associated with deploying isotope-specific imagers is beyond the scope of our paper. However it does seem plausible that since the scale of smuggling is so large, deployment of broadly capable detection systems could in effect pay for themselves in addition to strengthening national security. As well, the ability of these systems to detect common contraband may provide incentive for the DEA and Department of Customs to invest in projects important for the Domestic Nuclear Detection Office (DNDO).

\section{NRF Detection Systems: Performance and Key Metrics}

To set the stage for a discussion of contraband detection we give here a basic account of nuclear resonance fluorescence and the performance of NRF detection systems. Nuclear resonance fluorescence refers to the absorption and re-emission of photons by a nucleus. Each nucleus has a set of discrete energies at which it can absorb photons. Since no two separate isotopes exhibit the same pattern of resonances, the set of resonant excitation energies serves as a unique isotopic fingerprint. Most nuclei are characterized by hundreds or thousands of low lying transitions, but for practical purposes only the strongest photo-absorption resonances are important. Typical nuclei exhibit a few strong resonances with excitation energies less than a few MeV. Depending on the energy resolution of the detection system and light source, just one strong resonance is usually sufficient for uniquely identifying an isotope. Table 1 gives a list of some of the stronger resonances that have been found in isotopes interesting for contraband detection.

Bertozzi and Ledoux (2004) have proposed two qualitatively different approaches for exploiting nuclear resonance fluorescence to find isotopes hidden in well-shielded systems. In both a beam of gamma rays is shined into the inspected container. Some of the photons in this beam (typically a small fraction of the total) have just the right energy to resonantly excite the isotope suspected of being in the container.

The first approach for detecting the suspected isotope can be viewed as the nuclear analog of conventional atomic x-ray imaging. In this approach statements about the presence or absence 
of a material are made by measuring characteristics of the light beam transmitted through the container. If the suspected isotope is present in the container, then this transmitted beam will be markedly deficient in resonant photons and so will contain a shadow or "notch". The process is illustrated in figure 3 . This approach is sometimes called shadow imaging. Pruet et al. (2006) present a quantitative account of the performance of these systems.

The second detection approach relies on observation of photons emitted following decay of nuclei in the container that have been resonantly excited. This can be characterized as "looking for reflections" and is illustrated in figure 4. Compared to shadow imaging these reflection based systems have received relatively little attention in the published literature. Nonetheless, reflection imaging may be a better choice for some applications. Among these are applications where a rigorous understanding of error rates is not needed and applications involving interrogation of thin samples.

In our discussion of contraband we will present two quantities useful for determining how easy it is to detect a specific item. The first is called $W_{\min }$ and simply corresponds to the smallest linear dimension of a material embedded in a well-shielded cargo that can be quickly detected with transmission imaging. Here "quickly" corresponds to a scan time of 1 second with a light source characteristic of proposed laser/linac sources (Hartemann et al. 2005; Barty \& Hartemann 2004), while "wellshielded" roughly corresponds to a shielding of 200 $\mathrm{g} / \mathrm{cm}^{2}$ (or about $20 \mathrm{~cm}$ of lead). As an example, if $W_{\min }=1 \mathrm{~cm}$ for an item, then in 1 second a detection system could positively identify the presence of a $1 \mathrm{~cm}$ thick piece of that material in a packed cargo container.

The different physics governing reflection imaging suggests a different measure for these systems. It is called $\tau_{\text {shield }}$ and corresponds to the thickest optical depth of shielding through which a given material can be quickly detected. For most materials and the energies we are interested in here the relation between optical depth and areal density is $\tau \approx(W \times \rho) / 20\left(\mathrm{~g} / \mathrm{cm}^{2}\right)$. Here $W$ is the size of the shielding and $\rho$ is the density of the shielding material. As an example, if $\tau_{\text {shield }}=5$ for a material, then in one second the detection system could

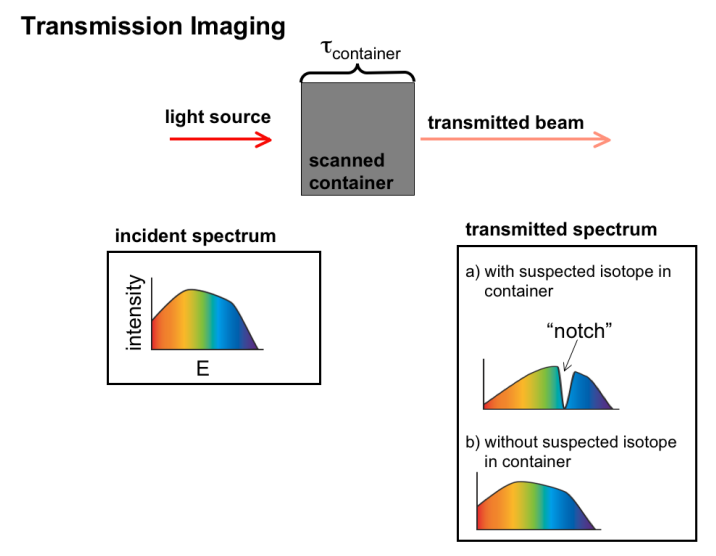

Fig. 3.- Illustration of a transmission-based detection system. For a more complete discussion of this type of system we refer the reader to Pruet et al. (2006).

\section{Reflection Imaging}

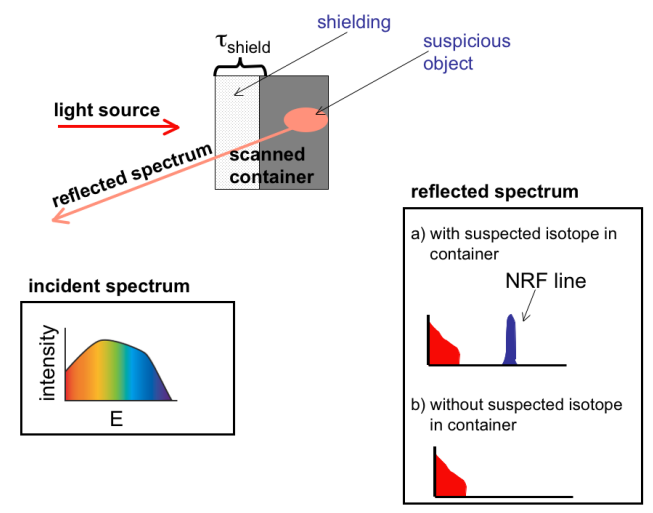

Fig. 4.- Illustration of a reflection-based detection system. Compared to transmission-based imaging systems these have received relatively little attention. Section 5.3 contains a basic account of the performance of these systems. 
TABLE 1

Some Experimentally Determined Resonances in Important Isotopes

\begin{tabular}{|c|c|c|}
\hline isotope $[\%]^{\mathrm{a}}$ & contraband category $^{\mathrm{b}}$ & prominent resonances $E\left[\sigma_{N R F}\right]^{c}$ \\
\hline${ }^{14} \mathrm{~N}[100]$ & drug & $2.31[0.16]$ \\
\hline${ }^{19} \mathrm{~F}[100]$ & $\mathrm{CW}, \mathrm{CFC}, \mathrm{EC}$ & $\begin{array}{l}1.55[1.80] \\
3.91[1.24]\end{array}$ \\
\hline${ }^{24} \mathrm{Mg}[79]$ & $\mathrm{EC}$ & $1.369[0.8]$ \\
\hline${ }^{31} \mathrm{P}[100]$ & $\mathrm{CW}, \mathrm{CSA}, \mathrm{EC}$ & $\begin{array}{r}1.27[1.12] \\
3.13[2.7]\end{array}$ \\
\hline${ }^{32} \mathrm{~S}[95]$ & $\mathrm{CW}, \mathrm{EC}$ & $2.23[1.62]$ \\
\hline${ }^{35} \mathrm{Cl}[76]$ & $\mathrm{CW}, \mathrm{CSA}, \mathrm{CFC}, \mathrm{EC}$ & $\begin{array}{l}1.22[1.16] \\
3.00[1.78]\end{array}$ \\
\hline${ }^{39} \mathrm{~K}[93]$ & CSA & $2.52[0.36]$ \\
\hline $\begin{array}{l}48 \mathrm{Ti}[74] \\
\left.{ }^{48} \mathrm{~T}\right]\end{array}$ & $\mathrm{EC}$ & $\begin{array}{l}3.370[1.2] \\
0.983[1.0]\end{array}$ \\
\hline${ }^{58} \mathrm{Ni}[68]$ & $\mathrm{PM}, \mathrm{EC}$ & $\begin{array}{l}3.263[2.0] \\
1.454[2.0]\end{array}$ \\
\hline${ }^{60} \mathrm{Ni}[26]$ & $\mathrm{PM}, \mathrm{EC}$ & $\begin{array}{l}1.332[1.9] \\
4.020[1.7]\end{array}$ \\
\hline${ }^{63} \mathrm{Cu}[69]$ & PM & $\begin{array}{l}0.669[7.1] \\
0.962[2.5]\end{array}$ \\
\hline${ }^{65} \mathrm{Cu}[31]$ & PM & $\begin{array}{l}0.770[9.5] \\
1.115[3.3]\end{array}$ \\
\hline${ }^{75} \mathrm{As}[100]$ & $\mathrm{CW}, \mathrm{EC}$ & $\begin{array}{c}1.07[3.49] \\
1.35[2.02]\end{array}$ \\
\hline${ }^{89} \mathrm{Y}[100]$ & $\mathrm{EC}$ & $\begin{array}{r}1.507[24.6] \\
2.882[4.2]\end{array}$ \\
\hline${ }^{90} \operatorname{Zr}[51]$ & $\mathrm{EC}$ & $\begin{array}{l}3.842[5.9] \\
2.186[5.5]\end{array}$ \\
\hline${ }^{93} \mathrm{Nb}[100]$ & $\mathrm{EC}$ & $\begin{array}{l}0.979[5.2] \\
0.744[3.5]\end{array}$ \\
\hline${ }^{92} \mathrm{Mo}[15]$ & $\mathrm{EC}$ & $3.945[8.3]$ \\
\hline${ }^{98} \mathrm{Mo}[24]$ & $\mathrm{EC}$ & $0.787[3.1]$ \\
\hline${ }^{101} \mathrm{Ru}[17]$ & PM & $0.720[1.8]$ \\
\hline${ }^{103} \mathrm{Rh}[100]$ & $\mathrm{PM}$ & $\begin{array}{r}0.803[12.2] \\
1.277[1.3]\end{array}$ \\
\hline${ }^{105} \operatorname{Pd}[22]$ & PM & $\begin{array}{l}0.442[4.5] \\
0.561[2.1]\end{array}$ \\
\hline${ }^{107} \mathrm{Ag}[52]$ & PM & $0.787[10.8]$ \\
\hline${ }^{109} \mathrm{Ag}[48]$ & PM & $0.701[9.6]$ \\
\hline${ }^{127} \mathrm{I}[100]$ & CSA & $1.04[3.2]$ \\
\hline${ }^{168} \operatorname{Er}[27]$ & $\mathrm{EC}$ & $1.79[53.8]$ \\
\hline${ }^{180} \mathrm{Hf}[35]$ & $\mathrm{EC}$ & $\begin{array}{l}2.582[15.5] \\
2.713[11.1]\end{array}$ \\
\hline${ }^{182} \mathrm{~W}[27]$ & $\mathrm{EC}$ & $\begin{array}{l}2.382[16.7] \\
2.474[11.4]\end{array}$ \\
\hline${ }^{184} \mathrm{~W}[31]$ & $\mathrm{EC}$ & $\begin{array}{r}0.903[2.9] \\
1.396[1.0]\end{array}$ \\
\hline${ }^{186} \mathrm{~W}[28]$ & $\mathrm{EC}$ & $0.738[1.9]$ \\
\hline${ }^{189} \mathrm{Os}[16]$ & PM & $0.531[35.4]$ \\
\hline${ }^{192} \mathrm{Os}[40]^{\mathrm{d}}$ & PM & $2.391[8.86]$ \\
\hline${ }^{196} \mathrm{Pt}[25]$ & PM & $2.824[11.5]$ \\
\hline${ }^{197} \mathrm{Au}[100]$ & PM & $0.503[9.7]$ \\
\hline${ }^{238} \mathrm{U}[99]$ & $\mathrm{EC}, \mathrm{SNM}$ & $\begin{array}{c}0.680[198] \\
1.782[17.0]\end{array}$ \\
\hline${ }^{239} \mathrm{Pu}[\text { unstable }]^{e}$ & EC,SNM & $\approx 2[\approx 10]$ \\
\hline
\end{tabular}

${ }^{a}$ The numbers in square brackets contain the fraction of the natural element comprised of the given isotope.

${ }^{\mathrm{b}}$ Class of contraband item that might be detected through detection of the listed element. The abbreviations are CW: chemical weapon, CFC: chlorofluorocarbon, CSA: chemical regulated under the controlled substances act, PM: precious metal, EC: export control and SNM: special nuclear material.

${ }^{c}$ Excitation energy of the in $\mathrm{MeV}$ and absorption cross section $\sigma_{N R F}$ in barns. Taken from NuDat (http://www.nndc.bnl.gov/nudat2/) and eq. 3.

d Taken from Fransen et al (1999).

${ }^{\text {e}}$ From preliminary analysis of very recent experimental data (Johnson 2006). 
identify the presence of the material as long as it is hidden behind less than about $10 \mathrm{~cm}$ of lead (or an equivalent shielding).

\section{Contraband Materials and Their De- tectability Through NRF}

Here we give an account of important contraband materials and the different isotopes comprising them. Information about prominent resonances in these isotopes is taken from the experimental data collected in the ENSDF database (ENSDF 2006). For a few isotopes the experimental survey of resonances is incomplete. Using the experimental resonance data and the formalism described in the appendices we estimate i) for transmission imaging the smallest quantity of each contraband item that can be efficiently detected in a well shielded container and ii) for reflection imaging the largest thickness of shielding through which the contraband item can be efficiently detected. Here "efficiently" means that interrogation takes less than one second. For transmission imaging a "well-shielded" container is one that represents 10 optical depths to photons of the resonance energy. It should be emphasized that we are assuming some nominal light source and detector characteristics and that for transmission with a laser/linac based light source. Readers interested in other detection system or shielding characteristics can refer to equations 9 and 11 in the appendices.

It is convenient to divide the discussion of contraband into different categories: chemical weapons, precious metals, export controlled items, drugs and chemicals regulated under the controlled substances act, fissile material and pollutants. Precious and fissile metals are identified uniquely by their isotopic content. Items like drugs and chemical weapons are special by virtue of their molecular structure. NRF is not sensitive to the low energy physics governing molecules. However, NRF is sensitive to specific isotopes that can serve as indicators or at least warning signs that a cargo is dangerous. For example, choking agents contain high concentrations of chlorine. Observation of this element in a barrel supposed to contain oil or grape juice would indicate foul play. In general, detection of chemical weapons or drugs through NRF has to rely on context-specific information like the cargo manifest or truck shipping statement.

\subsection{Chemical Weapons}

Chemical weapons are typically divided into classes based on their physiological effects. There are i) nerve agents, which inhibit transfer of nerve signals, ii) blister agents, which harm skin, eyes and mucous membranes, iii) blood agents, which prevent the body from using oxygen and iv) choking agents. We will see below that this division is also useful from the perspective of assessing NRFbased interrogation. All of these different chemical weapon types have been used for military or terrorist purposes. A full discussion of the different weapons, along with a historical account of their use, is given in the Fact-sheets on Chemical and Biological Warfare Agents (CBW Info 2006).

Table 2 gives a summary account of the detectability of well known chemical weapons. All of the agents that are stored as a liquid have one or two elements that are detectable in small samples and that might be an indication of the presence of a weapons. For example, we see from table 2 that both phosphorous and fluorine are detectable in samples of Sarin that have linear dimensions larger than about $15 \mathrm{~cm}$. Since very benign materials contain appreciable concentrations of both of these elements, their observation in a container would raise suspicions of a nerve agent. A more complete discussion of some different chemical weapons is given below.

\section{- Nerve Agents}

Most well-known nerve agents are stored as a liquid with a density near that of water and are comprised of the common elements $\mathrm{C}, \mathrm{N}$, $\mathrm{O}, \mathrm{H}$ and the less common fluorine, phosphorous and sulfur. Detection of nerve agents would rely on detection of phosphorous or phosphorous and fluorine (or phosphorous and sulfur for the VX agent) together. Table 1 contains a description of some of the useful resonances in the stable isotopes of fluorine, phosphorous and sulfur. All have resonances in the $\mathrm{MeV}$ range with thermal cross sections of one to three barns.

\section{GB (Sarin)}

This was used in the Tokyo subway attacks 
TABLE 2

Detectability of Common Chemical Weapons

\begin{tabular}{|c|c|c|c|c|c|}
\hline Agent & Class & Density $(\mathrm{g} / \mathrm{cc})^{\mathrm{a}}$ & Identifying Elements[\%] ${ }^{\mathrm{b}}$ & $W_{\min }(\mathrm{cm})^{\mathrm{c}}$ & $\tau_{\text {shield }}^{(\max ) \mathrm{d}}$ \\
\hline \multirow[t]{2}{*}{ GB(Sarin) } & nerve & 1.1 & $\mathrm{~F}[14]$ & 13.7 & 5.0 \\
\hline & & & $\mathrm{P}[22]$ & 9.4 & 5.3 \\
\hline \multirow[t]{2}{*}{ GA(Tabun) } & nerve & 1.1 & $\mathrm{~N}[17]$ & 94 & 4.4 \\
\hline & & & $\mathrm{P}[19]$ & 10.8 & 5.2 \\
\hline \multirow{2}{*}{ GD(Soman) } & nerve & 1.0 & $\mathrm{~N}[10]$ & 175 & 4.1 \\
\hline & & & $\mathrm{P}[17]$ & 13.3 & 5.1 \\
\hline \multirow{2}{*}{$\mathrm{GF}(\mathrm{Cyclosarin})$} & nerve & 1.1 & $\mathrm{~F}[10]$ & 19.9 & 4.8 \\
\hline & & & $\mathrm{P}[17]$ & 12.1 & 5.1 \\
\hline \multirow[t]{2}{*}{$\mathrm{VX}\left(\right.$ and $\left.\mathrm{VR}^{\mathrm{e}}\right)$} & nerve & 1.0 & $\mathrm{P}[12]$ & 18.9 & 5.0 \\
\hline & & & $\mathrm{S}[12]$ & 35 & 4.8 \\
\hline \multirow[t]{2}{*}{ CK(chlorine cyanide) } & blood & 1.2 & $\mathrm{Cl}[58]$ & 7.5 & 5.5 \\
\hline & & & $\mathrm{N}[23]$ & 63.7 & 4.6 \\
\hline \multirow[t]{2}{*}{ HD(sulfur mustard) } & blister & 1.3 & $\mathrm{Cl}[44]$ & 9.1 & 5.4 \\
\hline & blister & & $\mathrm{S}[20]$ & 16.0 & 5.0 \\
\hline \multirow[t]{2}{*}{ HN-1(a nitrogen mustard) } & blister & 1.1 & $\mathrm{~N}[8]$ & 200 & 4.0 \\
\hline & & & $\mathrm{Cl}[42]$ & 11.2 & 5.4 \\
\hline CG(phosgene) & choking & 1.4 & $\mathrm{Cl}[72]$ & 5.1 & 5.7 \\
\hline
\end{tabular}

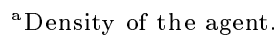

${ }^{\mathrm{b}}$ Elements in the agent that might be useful for identifying the weapon or for raising suspicion about a cargo. The fraction of the agent (by mass) comprised of the given element is given in square brackets.

${ }^{c}$ The smallest quantity (linear dimension) of the weapon in which the given element can be quickly identified through transmission imaging. Here quickly corresponds to a scan time of 1 second and it is assumed that the weapon is hidden in a well shielded environment with a total areal density near $200 \mathrm{~g} / \mathrm{cm}^{2}$.

${ }^{d}$ The largest optical depth of shielding through which the given element can be identified with reflection imaging. For photons with energies in the $\mathrm{MeV}$ range the corresponding areal density of the shielding is approximately $20 \times \tau_{\text {shield }} g / \mathrm{cm}^{2}$ for most materials. For example, a $\tau_{\text {shield }}$ of 5 corresponds to the ability to see through approximately $100 \mathrm{~g} / \mathrm{cm}^{2}$ with reflection imaging.

${ }^{e} \mathrm{VR}$ is a structural isomer of VX. 
by the Aum terrorist group and also by Iraq. Sarin is $13.5 \%$ fluorine by weight and $22 \%$ phosphorous by weight. Depending on the production method, it can be heavily contaminated with hydrogen fluoride. In order for the phosphorous to be detectable, the Sarin sample must have a linear dimension larger than about $9 \mathrm{~cm}$. For the fluorine in pure Sarin to be detectable the sample must have a linear size larger than about $14 \mathrm{~cm}$.

\section{GA (Tabun)}

Tabun is one of the easiest nerve agents to make and was a "starter" project for Iraq's weapons program. Tabun is $24 \%$ phosphorous by weight. In order for the phosphorous to be detectable, the Tabun must have a linear size larger than about $11 \mathrm{~cm}$. Tabun also has an appreciable nitrogen content. However, because of the lack of a strong low lying resonance in ${ }^{14} \mathrm{~N}$, the nitrogen can likely only be seen through reflection imaging.

\section{GD (Soman)}

This agent is more fatal than Sarin. It is comprised of $16 \%$ phosphorous by weight. Phosphorous in a sample of Soman with a linear dimension larger than $13 \mathrm{~cm}$ is detectable. The nitrogen in Soman can likely only be seen through reflection imaging.

\section{VX}

VX is relatively unique among the nerve agents in that it contains sulfur (12 percent by mass) as well as phosphorous (also 12 percent by mass). Because of the relatively low concentration of phosphorous, samples of VX must have a linear dimension larger than about $19 \mathrm{~cm}$ for the phosphorous to be detected. The sulfur is detectable in samples larger than about $35 \mathrm{~cm}$.

\section{- Blister agents}

Blister agents typically contain chlorine and are stored as a liquid with density near that of water. In addition to hydrogen and carbon, blister agents contain chlorine and either sulfur or nitrogen.

\section{HD ("mustard gas")}

HD contains $46 \%$ chlorine and $19 \%$ sulfur by weight. The chlorine is detectable in samples with linear dimensions larger than $9 \mathrm{~cm}$, the sulfur in samples with linear dimensions larger than about $16 \mathrm{~cm}$.

\section{Nitrogen Mustards}

These have a chlorine content similar to that of HD. The chlorine is detectable in samples with linear dimensions larger than about 11 $\mathrm{cm}$.

- Blood agents

\section{Ck (chlorine cyanide)}

This is $60 \%$ chlorine by weight and is a liquid with density $1.19 \mathrm{~g} / \mathrm{cc}$ at temperatures below 13.8 degrees $\mathrm{C}$. The chlorine is detectable in samples with linear dimensions larger than $8 \mathrm{~cm}$. The nitrogen in chlorine cyanide could be detected through transmission imaging in meter sized samples.

\section{SA (arsine)}

Though arsenic has a prominent resonance, this weapon is stored as a dilute gas. Only very large samples would be detectable with NRF.

\section{- Choking Agents}

The different choking agents are all rich in Chlorine. Chlorine gas is itself a choking agent but is difficult to detect with NRF because of the low density of the gaseous state. Of the more sophisticated choking agents, all can be transported as a liquid. Phosgene (CG) has the lowest boiling point (8 degrees Celsius) and is perhaps among the easiest to obtain and field. The chlorine in the different liquid choking agents is detectable in samples with linear dimensions larger than about $5 \mathrm{~cm}$.

\subsection{Precious Metals}

Metals with a high value per unit weight include gold, silver, platinum, osmium, rhodium, ruthenium, palladium. While gold, silver, and platinum are the most common of these, the other metals are significantly more valuable. In addition, we consider nickel and copper in this section, in part due to their recent increase in market price. These materials are used as money, jewelry, and have a wide variety of industrial uses including electroplating and metallurgy. These materials are 
TABle 3

Detectability of Precious Metals

\begin{tabular}{llll}
\hline \hline Material & Density $(\mathrm{g} / \mathrm{cc})$ & $W_{\min }(\mathrm{cm})^{\mathrm{a}}$ & $\tau_{\text {shield }}^{(\max ) \mathrm{b}}$ \\
\hline Copper & 8.933 & 1.4 & 5.4 \\
Gold & 19.282 & 0.21 & 5.6 \\
Nickel & 8.912 & 0.97 & 5.7 \\
Osmium & 22.57 & 0.19 & 5.8 \\
Platinum & 21.46 & 0.63 & 5.45 \\
Rhodium & 12.4 & 1.28 & 5.2 \\
Silver & 10.50 & 0.38 & 5.7 \\
\hline
\end{tabular}

${ }^{\mathrm{c}}$ The smallest quantity (linear dimension) of the metal that can be quickly identified through transmission imaging. Here quickly corresponds to a scan time of 1 second and it is assumed that the metal is hidden in a well shielded environment with a total areal density near 200 $\mathrm{g} / \mathrm{cm}^{2}$.

d The largest optical depth of shielding through which the metal can be identified with reflection imaging. For photons with energies in the $\mathrm{MeV}$ range the corresponding areal density of the shielding is approximately $20 \times$ $\tau_{\text {shield }} g / \mathrm{cm}^{2}$ for most materials. For example, a $\tau_{\text {shield }}$ of 5 corresponds to the ability to see through approximately $100 \mathrm{~g} / \mathrm{cm}^{2}$ with reflection imaging.

valued at between approximately 1 cent per gram (copper) to 100 dollars per gram (osmium).

The inherent value of these materials provides reason for smuggling. For example, the Ethiopian government estimates that revenue of more than $\$ 20$ million annually is lost due to gold smuggling. Purchasing gold is one method used to launder cash from drug sales and to finance terrorist activities (Farah 2005). Assets of the Taliban government frozen by the U.S. government in 1998 were held as gold reserves.

Additionally, more than 5000 kilograms of silver and nickel blocks were seized in 2005 between Hong Kong and mainland China. Similarly, the recent increase in commodity prices including copper and nickel has led to smuggling of coins, commonly made primarily of these metals.

Computers and consumer electronics are among the top commodities seized by U.S. Customs (FIA 2001). Also a method for money laundering, it is easy to imagine that a single truck or cargo container could a significant value in these goods. These commodities can potentially be identified by the presence of precious metals. For example, silver has the highest electrical conductivity of any metal, and thus is used for printed circuit boards in many electronics.
Table 3 shows the detectability of different metals. Most have multiple good NRF resonances and can be quickly detected in small quantities. An exception is gold, where the best known line for gold is at $502 \mathrm{keV}$. Photons of this low energy do not readily penetrate many materials. Relatively inexpensive experiments might discover strong resonances at higher energy in gold.

\subsection{Export controlled items}

Exports of some specialized goods out of the United States are regulated by the Export Administration Act of 1979. These controls are intended to enhance national security, foreign policy, nonproliferation, and limited supply interests of the United States. Items are regulated according to country of export and amount or frequency of export. Commerce controlled items are categorized in the Export Administration Regulations database into ten categories, including

- (0) Nuclear materials, facilities and equipment

- (1) Materials, chemicals, microorganisms, and toxins

- (2) Materials processing 
TABLE 4

Detectability of EXPorT CONTROL ITEMS

\begin{tabular}{|c|c|c|c|c|}
\hline Category $^{a}$ & Description & Identifying Element[\%] ${ }^{\mathrm{b}}$ & $W_{\min }(\mathrm{cm})^{c}$ & $\tau_{\text {shield }}^{(\max ) d}$ \\
\hline \multirow[t]{2}{*}{$0 \mathrm{C} 001$} & $\mathrm{U}, \mathrm{DU}, \mathrm{Th}$ & $\mathrm{U}[100]$ & 0.15 & 5.9 \\
\hline & & $\mathrm{Th}[100]$ & 1.8 & 5.0 \\
\hline $1 \mathrm{~A} 001$ & Materials made from fluorinated compounds & $\mathrm{F}$ & material dependent & \\
\hline \multirow{4}{*}{$1 \mathrm{C} 002^{\mathrm{e}}$} & Metal alloys & $\mathrm{Ti}[75]$ & 0.28 & 6.0 \\
\hline & & $\mathrm{Ni}[50]$ & 8.0 & 4.7 \\
\hline & & $\mathrm{Nb}[30]$ & 9.1 & 4.6 \\
\hline & & $\mathrm{Mg}[30]$ & 4.5 & 4.9 \\
\hline \multirow[t]{5}{*}{$1 \mathrm{C} 004^{\mathrm{e}}$} & $\mathrm{U}+\mathrm{Ti}, \mathrm{W}+\mathrm{Ni}+\mathrm{Cu}$ alloys & $\mathrm{U}[80]$ & 0.6 & 5.8 \\
\hline & & $\mathrm{Ti}[20]$ & 1.0 & 5.3 \\
\hline & & $\mathrm{W}[40]$ & 3.4 & 5.1 \\
\hline & & $\mathrm{Ni}[30]$ & 13.2 & 4.4 \\
\hline & & $\mathrm{Cu}[30]$ & 0.67 & 5.5 \\
\hline \multirow[t]{2}{*}{$1 \mathrm{C} 006$} & Lubricating materials (Chlorofluorocarbons) & $\mathrm{F}[30]$ & 7.0 & 5.4 \\
\hline & & $\mathrm{Cl}[30]$ & 17.3 & 5.2 \\
\hline \multirow[t]{2}{*}{$1 \mathrm{C} 011^{\mathrm{e}}$} & Metals and compounds & $\operatorname{Zr}[100]$ & 1.0 & 5.8 \\
\hline & & $\mathrm{Mg}[100]$ & 1.4 & 5.5 \\
\hline \multirow[t]{2}{*}{$1 \mathrm{C} 111^{\mathrm{e}}$} & Propellants & $\operatorname{Zr}[97]$ & 1.0 & 5.8 \\
\hline & & $\operatorname{Mg}[97]$ & 1.4 & 5.5 \\
\hline \multirow[t]{2}{*}{$1 \mathrm{C} 117^{\mathrm{e}}$} & Rocket motor components & $\operatorname{Mo}[100]$ & 2.5 & 5.5 \\
\hline & $\mathrm{W}[100]$ & 1.4 & 5.6 & \\
\hline \multirow[t]{4}{*}{$2 \mathrm{~A} 225^{\mathrm{e}}$} & Crucibles resistant to liquid actinide materials & $\operatorname{Zr}[74]$ & 2.5 & 5.4 \\
\hline & & $Y[65]$ & & \\
\hline & & $\operatorname{Er}[87]$ & 0.19 & 5.9 \\
\hline & & $\mathrm{Ta}[100]$ & 0.44 & 5.9 \\
\hline
\end{tabular}

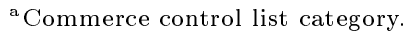

${ }^{b}$ Elements in the agent that might be useful for identifying the item or for raising suspicion about a cargo. A rough estimate of the fraction of the item (by mass) comprised of the given element is given in square brackets.

${ }^{c}$ The smallest quantity (linear dimension) of the item in which the given element can be quickly identified through transmission imaging. Here quickly corresponds to a scan time of 1 second and it is assumed that the item is hidden in a well shielded environment with a total areal density near $200 \mathrm{~g} / \mathrm{cm}^{2}$.

d The largest optical depth of shielding through which the given element can be identified with reflection imaging. For photons with energies in the $\mathrm{MeV}$ range the corresponding areal density of the shielding is approximately $20 \times \tau_{\text {shield }} g / \mathrm{cm}^{2}$ for most materials. For example, a $\tau_{\text {shield }}$ of 5 corresponds to the ability to see through approximately $100 \mathrm{~g} / \mathrm{cm}^{2}$ with reflection imaging.

${ }^{e}$ For these categories we have assumed materials to have a density of $6 \mathrm{~g} / \mathrm{cc}$. 
Table 4 lists some of the characteristic materials from these categories and their NRF detection characteristics. Some controlled chemicals are not listed here, and instead are described in the following section under the Controlled Substances Act.

While the detailed characteristics (for example: material purity, designed use) that make items export controlled are not detectable, the detection of these materials when not indicated by manifest data would be a clear warning sign. In addition, many of these materials are not found in typical cargo, so observing them via their NRF signature may in some cases be cause enough for further investigation.

\subsection{Drugs and Chemicals Regulated Un- der the Controlled Substances Act}

Synthesis of cocaine, heroin and other common drugs requires large quantities of chemicals. In an attempt to hinder illicit drug production, particularly by South American countries, the U.S. (through the controlled substances act) and the U.N. (through article 12) regulate trade in chemicals key to drug processing. There are presently thirty eight chemicals regulated under the controlled substances act.

Most of the regulated chemicals seem either difficult to detect with NRF or comprised of elements that are too common to raise suspicion. However, a few contain substantial quantities of potentially tell-tale elements. A brief discussion of some of these is given below, while a summary of the different regulated chemicals is given in table 5 .

\section{- Phosphorous}

Phosphorous is a reagent used in the production of amphetamines and methamphetamines. Trade of any quantity of pure phosphorous is regulated (no minimum regulated quantity is specified), though many unregulated substances also contain phosphorous. Different states of phosphorous (red, yellow and white) have a density near 2 $\mathrm{g} / \mathrm{cc}$. As discussed in the chemical weapons section, phosphorous has pronounced resonances that allow rapid detection.

\section{- Potassium Permanganate}

Potassium permanganate is a reagent used in cocaine production. International trade of more than $500 \mathrm{~kg}$ is regulated. This chemical is $25 \%$ potassium and $35 \%$ manganese by weight. It has a density of $2.7 \mathrm{~g} / \mathrm{cc}$. The potassium and manganese in small quantities of potassium permanganate can be quickly detected.

\section{- Sulfuric acid}

Sulfuric acid is a reagent used in the production of many drugs including cocaine and methamphetamines. Export of quantities larger than $247 \mathrm{~kg}$ is regulated. Sulfuric acid is $33 \%$ sulfur by mass and has a density of $1.84 \mathrm{~g} / \mathrm{cc}$. The sulfur in small quantities of sulfuric acid can be quickly detected.

\section{- Iodine}

Iodine is a reagent used in amphetamine and methamphetamine production and has a density near $5 \mathrm{~g} / \mathrm{cc}$. Domestic trade of more than $400 \mathrm{~g}$ is regulated. Because of the existence of a strong resonance in iodine, small quantities of this element can be quickly detected.

Detection of drugs themselves through NRF might be accomplished through detection of nitrogen. From the view of elemental composition, nitrogen marks the difference between many innocuous substances like sugar and alcohol and illicit drugs. Common drugs like cocaine, heroine and methamphetamines are comprised of a few percent nitrogen by weight. Because of the paucity of nitrogen and the lack of a strong resonance in ${ }^{14} \mathrm{~N}$, detection of nitrogen through transmission imaging is only possible for very large (linear dimensions of order a meter) samples. Detection of nitrogen through reflection imaging is still possible for modestly shielded cargos. Table 6 summarizes the detectability of nitrogen in different common drugs.

\subsection{Fissile Materials}

The explosion energy in nuclear weapons is generated through the fission of heavy nuclei. There are a handful of so-called fissile isotopes with large cross sections for fission induced by low energy neutrons. The most important of these are ${ }^{239} \mathrm{Pu}$ and ${ }^{235} \mathrm{U}$. Plutonium is not stable and so must be synthesized in reactors. Uranium 235 is stable and it's present day inventory can be traced to 
TABLE 5

Detectability of Some Chemicals Regulated Under the Controlled Substances Acta

\begin{tabular}{lllll}
\hline \hline \multicolumn{1}{c}{ Chemical } & Regulated Quantity ${ }^{\mathrm{b}}(\mathrm{kg})$ & Identifying Elements[\%] $^{\mathrm{c}}$ & $W_{\min }(\mathrm{cm}){ }^{\mathrm{d}} \tau_{\text {shield }}^{(\mathrm{max}) \mathrm{e}}$ \\
\hline Phosphorous & 0 & $\mathrm{P}[100]$ & 1.1 & 6.0 \\
Potassium Permanganate & 500 & $\mathrm{~K}[25]$ & 5.6 & 5.2 \\
& & $\mathrm{Mn}[35]$ & 0.6 & 5.7 \\
Sulfuric Acid & 247 & $\mathrm{~S}[33]$ & 6.9 & 5.3 \\
Iodine & 0.4 & $\mathrm{I}[100]$ & 1.6 & 5.5 \\
\hline
\end{tabular}

a Taken from the U.S. drug enforcement agency (dea.gov).

${ }^{\mathrm{b}}$ Threshold quantity of the chemical triggering regulation.

${ }^{\mathrm{c}}$ Elements that might be useful for identifying the regulated chemical. The fraction of the chemical (by mass) comprised of the given element is given in square brackets.

${ }^{\mathrm{d}}$ The smallest quantity (linear dimension) of the substance in which the given element can be quickly identified through transmission imaging. Here quickly corresponds to a scan time of 1 second and it is assumed that the chemical is hidden in a well shielded environment with a total areal density near $200 \mathrm{~g} / \mathrm{cm}^{2}$.

eThe largest optical depth of shielding through which the given element can be identified with reflection imaging. For photons with energies in the $\mathrm{MeV}$ range the corresponding areal density of the shielding is approximately $20 \times \tau_{\text {shield }} g / \mathrm{cm}^{2}$ for most materials. For example, a $\tau_{\text {shield }}$ of 5 corresponds to the ability to see through approximately $100 \mathrm{~g} / \mathrm{cm}^{2}$ with reflection imaging.

TABLE 6

Detectability of Nitrogen in Some Commonly Trafficked Drugs ${ }^{a}$

\begin{tabular}{lll}
\hline \hline \multicolumn{1}{c}{ Drug } & Percent Nitrogen By Weight & $\tau_{\text {shield }}^{(\max )}$ \\
\hline Heroin & 3.8 & 3.7 \\
Cocaine & 4.6 & 3.8 \\
Methamphetamine & 9.4 & 4.1 \\
\hline
\end{tabular}

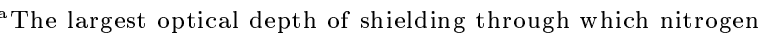
in the drug can be identified with reflection imaging. For photons with energies in the $\mathrm{MeV}$ range the corresponding areal density of the shielding is approximately $20 \times \tau_{\text {shield }} \mathrm{g} / \mathrm{cm}^{2}$ for most materials. For example, a $\tau_{\text {shield }}$ of 5 corresponds to the ability to see through approximately $100 \mathrm{~g} / \mathrm{cm}^{2}$ with reflection imaging. Because of the small concentration of Nitrogen in the different drugs and because of the absence of a very strong low lying resonance in Nitrogen, this element is not detectable through transmission imaging except in very large samples. 
stellar explosions, but only a small fraction of natural uranium is comprised of the 235 isotope while Uranium-238 comprises the lion's share. For this reason uranium that is enriched in the 235 isotope always has some concentration of ${ }^{238} \mathrm{U}$.

The experimental study of resonances in uranium and plutonium is still incomplete. Uranium 238 is relatively well studied and several strong resonances have been found. In particular, there is a strong resonance line at an excitation energy of $1846 \mathrm{keV}$ that has an effective thermal cross section near 20 barns. As a result rather small quantities of this isotope can be quickly found through $\mathrm{NRF}, W_{\min } \approx 0.2 \mathrm{~cm}$. Very recently experiments have been done at the MIT HVRL facility to study ${ }^{235} \mathrm{U}$ and ${ }^{239} \mathrm{Pu}$. Initial analysis of the ${ }^{239} \mathrm{Pu}$ experiments indicates the present of a line with an absorption cross section near 10 barns at an excitation energy near $2 \mathrm{MeV}$ (Johnson 2006). This would imply that quantities of $\mathrm{Pu}$ as small as $W_{\min } \approx 0.3 \mathrm{~cm}$ could be quickly found in well shielded containers. Again, though, we caution that these are tentative results. Results of the ${ }^{235} \mathrm{U}$ experiments are not yet publicly available.

\subsection{Pollutants (chlorofluorocarbons)}

The 1987 Montreal Protocol formalized an agreement between developed nations to dramatically reduce the use of chemicals that deplete the ozone layer. Among the most harmful of these are chlorofluorocarbons (CFC's). Because these have useful applications, particularly for automobile air conditioning, a vibrant illicit trade in CFC's began after the Montreal Protocol. It is estimated that roughly 2,000 tons of CFC's worth about $\$ 100 \mathrm{M}$ are smuggled into the U.S. each year.

CFC's are composed of hydrogen, carbon, chlorine and fluorine. CFC-12 (freon) is $31 \%$ fluorine and $52 \%$ chlorine by weight. In pressurized containers CFC's are found in a liquid state. Estimates of the quantity and the maximum amount of shielding through which CFC's can be quickly detected are shown in table 7. Rapid detection of fluorine and chlorine is possible for even small quantities of CFC's.

\section{Conclusions}

NRF detection systems could effectively provide an "elemental manifest" containing a detailed account of many different elements present in a cargo. With proposed laser/linac light sources the scan time needed to generate this manifest would be rather short, of order seconds even for well shielded cargos. Here we have considered the detectability of a few different types of contraband including chemical weapons, chemicals regulated under the controlled substances act, precious metals, fissile materials and some items regulated under export control laws. It was found that NRF systems could be used to find items in all of these categories. We have not exhaustively considered possible customs applications, and have entirely neglected some potentially fruitful applications such as detecting tariff avoidance. Since NRF systems are capable of detecting a wide variety of potential contraband they may be a useful tool in the substantial fight against smuggling as well as for efforts to prevent domestic nuclear attacks.

We thank Dennis McNabb, Fred Hartemann and Rob Schmidt for insightful discussions.

This work was performed under the auspices of the U.S. Department of Energy by University of California, Lawrence Livermore National Laboratory under Contract W-7405-Eng-48.

\section{REFERENCES}

Barty, C.P.J. \& Hartemann, F.V. 2004, Lawrence Livermore Laboratory Report, UCRL-TR206825

Bauske et al. 1993, Phys. Rev. Lett. 71, 975

Bertozzi, W. \& Ledoux, B. 2004, CAARI proceeding

Chemical Weapons Fact-sheets http://cbwinfo.com

Environmental Investigation Agency, 2003, "Lost In Transit: Global CFC Smuggling Trends and the Need for a Faster Phase-Out"

Evaluated Nuclear Structure Data Files http://www.nndc.bnl.gov/ensdf.

Testimony of Douglas Farah (Feb. 16, 2005) "Terrorist Responses to Improved U.S. Financial Defences" before the House Subcommittee on Oversight and Investigations. 
TABle 7

Detectability of Freon (and similar ${ }^{a}$ ) Chlorofluorocarbons regulated under the Montreal Protocol

\begin{tabular}{clll}
\hline \hline Name & Identifying Elements[\%] $^{\mathrm{b}}$ & $W_{\min }(\mathrm{cm})^{\mathrm{c}}$ & $\tau_{\text {shield }}^{(\max ) \mathrm{d}}$ \\
\hline CFC-12 (Freon) & $\mathrm{Cl}[52]$ & 10.0 & 5.5 \\
& $\mathrm{~F}[31]$ & 6.8 & 5.4 \\
\hline
\end{tabular}

\footnotetext{
a By definition the different CFC's have high concentrations of fluorine and chlorine and are roughly equivalent as far as detection through NRF is concerned.

${ }^{\mathrm{b}}$ Elements in the agent that might be useful for identifying CFC's. The fraction of Freon (by mass) comprised of the given element is given in square brackets.

${ }^{\mathrm{c}}$ The smallest quantity (linear dimension) of Freon in which the given element can be quickly identified through transmission imaging. Here quickly corresponds to a scan time of 1 second and it is assumed that the freon is hidden in a well shielded environment with a total areal density near $200 \mathrm{~g} / \mathrm{cm}^{2}$.

${ }^{\mathrm{d}}$ The largest optical depth of shielding through which the given element can be identified with reflection imaging. For photons with energies in the $\mathrm{MeV}$ range the corresponding areal density of the shielding is approximately $20 \times \tau_{\text {shield }} g / \mathrm{cm}^{2}$ for most materials. For example, a $\tau_{\text {shield }}$ of 5 corresponds to the ability to see through approximately $100 \mathrm{~g} / \mathrm{cm}^{2}$ with reflection imaging.
}

FIA International Research, 2001, "Contraband, Organized Crime and the Threat to the Transportation and Supply Chain Function"

Fransen, C. et al. 1999, Phys. Rev. C, 59, 2644

Hamilton, W.D., Ed. 1975, "Electromagnetic Interactions in Nuclear Spectroscopy", NY, American Elsevier, Amsterdam

Hartemann, F.V., Brown, W.J., Gibson, D.J., Anderson, S.G., Tremaine, A.M., Springer, P.T., Wootton, A.J., Hartouni, E.P. \& Barty, C.P.J. 2005, Phys. Rev. ST Accel. Beams 8, 100702

Johnson, M. 2006, private communication

Kneissl, U., Pitz, H.H. \& Zilges, A. 1996, Prog. Part. Nucl. Phys., 37, 349

Margraf, J. Degener, A., Friedrichs, H., Heil, R. D., Jung, A., Kneissl, U., Lindenstruth, S., Pitz, H. H., Schacht, H., Seemann, U., Stock, R. \& Wesselborg, C. 1990, Phys. Rev. C 42, 771

Metzger, F.R. 1956, Phys. Review, 101, 286

McNabb, D. \& Pruet, J. 2005, UCRL-TR-210052 (OUO, request copy from $\mathrm{D}$. McNabb)
Pruet, J., McNabb, D.M., Hagmann, C.Y., Hartemann, F.V. \& Barty, C.P.J., 2006, jo. of Appl. Physics, 99, 123102

Schiff, L. I. 1946, Phys. Rev. 70, 761

\section{Appendices}

\subsection{The NRF cross section}

An introduction to the theory behind nuclear resonance fluorescence and to experimental studies of this process can be found in Kneissl, Pitz \& Zilges (1996). For our purposes we need to know the relation between experimentally-measured nuclear properties and the cross section for nuclear photo-absorption. The peak cross section for a nucleus in state $i$ to capture a photon and be directly excited to state $j$ is

$\sigma_{\gamma}\left(E=E_{\text {res }}\right)=2.5 \cdot 10^{3} \mathrm{~b}\left(\frac{1 \mathrm{MeV}}{E_{\text {res }}}\right)^{2}\left(\frac{2 J_{j}+1}{2 J_{i}+1}\right)$.

Here $J$ denotes spin and $E_{\text {res }}$ represents the excitation energy of the state $i$ relative to the state

This 2-column preprint was prepared with the AAS IATEX macros v5.0. 
$j$. Atoms in material at finite temperature have non-zero velocities. As a result, the absorption cross section is thermally broadened and has an effective width

$$
\Gamma_{\text {thermal }} \approx 0.5 \mathrm{eV} \sqrt{\frac{240}{A}}\left(\frac{E_{\mathrm{res}}}{1 \mathrm{MeV}}\right) .
$$

Here we have assumed that the intrinsic width $\Gamma_{0}$ describing decay of the excited state to the ground state is much smaller than $1 \mathrm{eV}$. In this case the thermal average of the resonant scattering cross section is approximately

$$
\begin{aligned}
\sigma_{\mathrm{NRF}} & \approx 0.75 \sigma_{\gamma} \frac{\Gamma_{0}}{\Gamma_{\text {thermal }}} \\
& =3.75 \text { barns }\left(\frac{1 \mathrm{MeV}}{E}\right)^{3}\left(\frac{\Gamma_{0}}{1 \mathrm{meV}}\right) \\
& \times\left(\frac{2 J_{j}+1}{2 J_{i}+1}\right) \sqrt{\frac{A}{240}}
\end{aligned}
$$

Here the prefactor 0.75 comes from a consideration of the Maxwellian distribution of atom velocities in the sample (Metzger 1956). Experiments often quote the lifetime $\tau$ of a state rather than the width. The two are related by $\Gamma=\hbar / \tau$, or

$$
\Gamma \approx 0.66 \mathrm{meV}\left(\frac{1 \mathrm{ps}}{\tau}\right)
$$

The lifetime $\tau$ and the half life $\tau_{1 / 2}$ of the state are related through $\tau_{1 / 2}=\tau \ln (2)$.

\subsection{Shadow Imaging}

Detection of an isotope with shadow imaging relies on developing a healthy "notch" in the spectrum transmitted through cargo. For this reason the time needed to find an isotope in a material sample depends on $\tau_{\text {res }}$, the optical depth of the sample to resonant absorption. If the sample is optically thick to resonant absorption, then essentially all resonant photons that are not scattered by atomic processes will excite nuclei. If the sample is thin, then photons are not efficiently absorbed by nuclear processes and shadow detection is difficult.

In terms of the NRF cross section, the optical depth of a material to resonant scattering is

$$
\begin{aligned}
\tau_{\mathrm{NRF}} & =\sigma_{\mathrm{NRF}} \rho_{\mathrm{p}} W N_{A} / A \\
& \approx 0.06\left(\frac{\sigma_{\mathrm{NRF}}}{10 \mathrm{barns}}\right)\left(\frac{\rho_{\mathrm{p}} W}{1 \mathrm{~g} / \mathrm{cm}^{2}}\right)\left(\frac{100}{A}\right)(8)
\end{aligned}
$$

Here $\rho_{\mathrm{p}}$ is the partial density of the isotope we're looking for, $A$ is the atomic number of the isotope and $W$ is the linear dimension characterizing the interrogated sample. The subscript $\mathrm{p}$ on the density is used to emphasize that the relevant density is the partial density of the isotope, not the entire density of the material.

The time taken to detect a particular isotope with shadow imaging was worked out in Pruet et al. (2006):

$$
t_{\text {scan }}=\frac{36 \exp \left(\tau_{\text {container }}\right)}{\epsilon_{D} \phi_{\text {beam }} \Gamma_{\text {thermal }}}\left(\frac{1}{1-\exp \left(-\tau_{\text {res }}\right)}\right)^{2} .
$$

Here $\tau_{\text {container }}$ is the optical depth of the container to off-resonant photons, $\epsilon_{D}$ is the efficiency of the detector used to observe resonant photons in the beam escaping the container and $\phi_{\text {beam }}$ is the $\mathrm{flu}$ ence (photons / unit time $\times$ unit energy) characterizing the beam. Note that apart from a modest dependence on the thermal absorption width, the only dependence on the properties of the suspected isotope is in the term containing $\tau_{\text {res }}$.

Figure 5 shows the time taken to detect the presence of a particular isotope as a function of the resonant optical depth of the scanned material. For this figure we have assumed a light source with $\phi_{\text {beam }}=10^{15} /(\sec \times \mathrm{MeV})$. This is thought to be characteristic of next generation sources that generate high energy photons by scattering laser light from energetic electrons (Hartemann et al. 2005). The first generation of these systems are expected to be about one percent as bright. For the detector we assumed $\epsilon_{D}=0.1$ and for the resonance we assumed $\Gamma_{\text {thermal }}=1 \mathrm{eV}$. The optical depth of the scanned cargo has been taken to be $\tau_{\text {container }}=10$.

It is seen from figure 5 that samples which are thick to resonant absorption can be quickly detected. Detection of thin samples $\left(\tau_{\text {res }}<1\right)$ is more difficult, with the scan time scaling as $1 / \tau_{\text {res }}^{2}$ in the thin limit. For the sake of adopting a convention we will suppose that the typical container has an optical depth of 10 (for purely atomic processes) to photons with the resonant energy. For $E_{\text {res }}$ in the range of one to a few $\mathrm{MeV}$ this corresponds to an areal density $(\rho \times W)$ of approximately $200 \mathrm{~g} / \mathrm{cm}^{2}$. We will also suppose that the system should be able to detect the presence of an isotope in one second. These conventions allow the 
definition of the minimum linear dimension of material that is detectable with transmission imaging

$$
W_{\min }=2 \mathrm{~cm}\left(\frac{10 \text { barns }}{\sigma_{\mathrm{NRF}}}\right)\left(\frac{1 \mathrm{~g} / \mathrm{cc}}{\rho_{p}}\right)\left(\frac{A}{100}\right) .
$$

This minimum detectable size corresponds to $\tau_{\text {res }} \approx 0.1$.

\subsection{Reflection Imaging}

Compared to shadow imaging, this type of system is relatively complicated and not well studied. Still, the basic considerations governing detection can be readily worked out. Possibly the only important unknown relates to processes that give rise to high energy backgrounds in the detector observing resonantly emitted photons. For the present purposes we will ignore these backgrounds. This is thought to be a fair assumption for systems using detectors and light sources with fine energy resolution.

A rough estimate for the time needed to identify the presence of an isotope in a sample with reflection imaging is given by

$$
t_{\text {scan }}=36 \frac{\sigma_{\text {tot }}}{\sigma_{\mathrm{NRF}}} \frac{\exp \left(2 \tau_{\text {shield }}\right)}{\epsilon_{D} \phi_{\text {beam }} \Gamma_{\text {thermal }}} .
$$

Here $\sigma_{\text {tot }} / \sigma_{\mathrm{NRF}}$ is the ratio of the total photon interaction cross section for the interrogated material to the NRF cross section for that material, $\tau_{\text {shield }}$ is the optical depth of any material shielding the sample, and other symbols have the same meaning as in eq. 9. The prefactor of 2 multiplying $\tau_{\text {shield }}$ arises because resonant photons have to get both in and out of the shielding material. The above equation assumes that the interrogating material is optically thick to atomic scatterings at the resonance energy. This is satisfied for almost all interesting sized samples (e.g. those with areal densities $\rho W>20 \mathrm{~g} / \mathrm{cm}^{2}$ ). Figure 4 gives an illustration of a reflection system.

For reflection imaging we adopt a different figure of merit than for transmission imaging. We will specify the largest thickness of shielding $\left(\tau_{\text {shield }}\right)$ through which an isotope in a sample can be quickly identified. Here again we suppose that "quickly" corresponds to an interrogation time of 1 second. From eq. 11 we have

$$
\tau_{\text {shield }} \approx 6.3+\frac{1}{2} \ln \left(\frac{\sigma_{\mathrm{NRF}}}{\sigma_{\text {tot }}}\right) .
$$

For this equation characteristics of the interrogation beam were assumed to be the same as used in generating figure 5 . For the detection system we assumed an efficiency (that includes the solid angle) of $1 \%$ rather than the $10 \%$ efficiency assumed for the transmission detection system. This is because in the transmission system the detector only has to observe photons scattered from a small sample foil, while in the reflection system the detector must observe photons originating from a relatively large volume. As an example of eq. 12, an isotope for which $\sigma_{\mathrm{NRF}} / \sigma_{\mathrm{tot}}=0.1$ can be detected in less than a second as long as it is shielded by material representing less than 5 optical depths to resonant photons. This corresponds to about $100 \mathrm{~g} / \mathrm{cm}^{2}$ for photons with an energy in the one to few $\mathrm{MeV}$ range. 


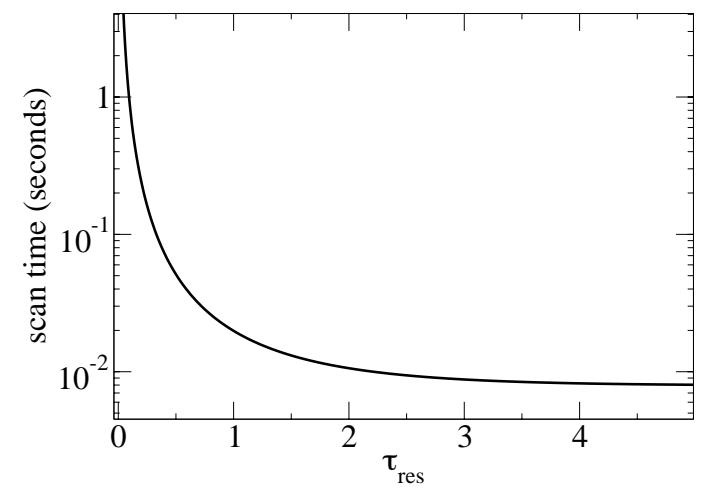

Fig. 5.- Time needed to detect a specific isotope within a cargo using transmission imaging as a function of the optical depth of that cargo to nuclear resonant absorption. For this figure the optical depth of the cargo to atomic processes is assumed to be 10, which corresponds to an areal density of about $200 \mathrm{~g} / \mathrm{cm}^{2}$. Characteristics of the interrogating light source are those estimated for next generation laser/linac beams. See text for details. 